\title{
DEEP LEARNING FOR FRAME ERROR PROBABILITY PREDICTION IN BICM-OFDM SYSTEMS
}

\author{
Vidit Saxena $^{1,2}$, Joakim Jaldén ${ }^{1}$, Mats Bengtsson ${ }^{1}$, and Hugo Tullberg ${ }^{2}$ \\ ${ }^{1}$ Department of Information Science and Engineering, KTH, Stockholm \\ ${ }^{2}$ Ericsson Research, Stockholm
}

\begin{abstract}
In the context of wireless communications, we propose a deep learning approach to learn the mapping from the instantaneous state of a frequency selective fading channel to the corresponding frame error probability (FEP) for an arbitrary set of transmission parameters. We propose an abstract model of a bit interleaved coded modulation (BICM) orthogonal frequency division multiplexing (OFDM) link chain and show that the maximum likelihood (ML) estimator of the model parameters estimates the true FEP distribution. Further, we exploit deep neural networks as a general purpose tool to implement our model and propose a training scheme for which, even while training with the binary frame error events (i.e., ACKs / NACKs), the network outputs converge to the FEP conditioned on the input channel state. We provide simulation results that demonstrate gains in the FEP prediction accuracy with our approach as compared to the traditional effective exponential SIR metric (EESM) approach for a range of channel code rates, and show that these gains can be exploited to increase the link throughput.
\end{abstract}

Index Terms - FEP, BICM-OFDM, Deep Learning, Neural Networks, MCS Selection, Fast Link Adaptation.

\section{INTRODUCTION}

The efficiency of a radio link depends on its ability to adapt to the stochastic radio channel conditions that typically vary over time (i.e., fading) as well as over the signal bandwidth (i.e., frequency selectivity. Practical radio systems solve this problem by selecting the transmission parameters in each frame to fulfil some optimality criteria such as desired throughput, latency, etc. [1]. In this paper we investigate the problem of frame error probability (FEP) prediction for bit-interleaved coded modulation orthogonal frequency division multiplexing (BICM-OFDM) systems [2] [3], which is an essential step in the selection of optimal tranmission parameters. Owing to their flexibility and performance, BICM-OFDM systems have been widely adopted by most of the modern radio air interfaces including those for local wireless area networks (e.g., WiFi) and for cellular communication such as Long Term Evolution for $4 \mathrm{G}$, and more recently, New Radio for 5G [4].

In general for frequency selective channels, it is intractable to compute the FEP conditioned on the frame channel state characterized by the received per-subcarrier signal to interference and noise ratios (SINRs). Therefore, several approximate teachniques for FEP prediction have been developed that compress the channel state vector to an approximate effective channel state scalar, which is mapped to pre-computed FEP values stored within lookup tables [5] [6] [7].

This work was partially supported by the Wallenberg Autonomous Systems and Software Program (WASP).
In addition to the loss of channel state information in this approach, the choice of compression function is somewhat arbitrary and the optimality of the predicted FEP has only been studied empirically [8].

As an alternative to the effective SINR approach, supervised learning techniques that directly learn the mapping between persubcarrier SINRs and the corresponding FEP have been developed recently [9] [10]. In the traning phase of these techniques, the persubcarrier SINRs for several channel realizations along with their Monte Carlo simulated FEPs are used to iteratively train the model parameters until some convergence criteria is satisfied. With sufficient training, these techniques have been shown to improve the realized link throughput in BICM-OFDM systems compared to the effective SINR approach. However, these techniques do not provide any insight into the optimality of the trained models.

In this paper, we cast the FEP prediction problem as a probabilistic binary classification task, where the classes correspond to frame error and success events (i.e., NACKs and ACKs) respectively, and make the following three main contributions: (i) We propose an abstract model of the BICM-OFDM link chain where the observations are the frame channel states and their binary frame error events and show that, in the limit of infinite training samples, the maximum likelihood (ML) estimator of the model parameters estimates the true FEP distribution, (ii) we use this model to develop a supervised learning approach for FEP prediction based on deep neural networks, where the training phase requires only the observed channel states and binary frame error events, thus eliminating the need for tedious Monte Carlo simulations to measure the FEP, and (iii) we provide numerical results to show that our approach improves the FEP prediction accuracy compared to one widely studied effective SINR approach, and exploit these predictions to improve the realized link throughput.

The rest of this paper is organized as follows: In Sec. 2 we describe a typical BICM-OFDM wireless communication link and propose an abstract system model along with an ML estimator of the model parameters. Next in Sec. 3, we summarize a commonly studied effective SINR approach for FEP prediction and introduce our deep learning approach. Finally in Sec. 4 we present simulation results for FEP prediction accuracy and realized throughput for the two considered approaches and in Sec.5 we conclude the paper.

\section{BICM-OFDM SYSTEM AND ML ESTIMATION}

\subsection{System Model}

We consider a BICM-OFDM link chain similar to the LTE downlink and illustrate its block diagram in Fig. 1 Here, a "transport block", $\boldsymbol{b}=\left(b_{1}, \ldots, b_{T}\right)$ of information bits is first encoded by a channel encoder and subsequently bit-interleaved by a random interleaver to generate the bit sequence $\boldsymbol{l}=\left(l_{1}, \ldots, l_{L}\right)$. The inter- 


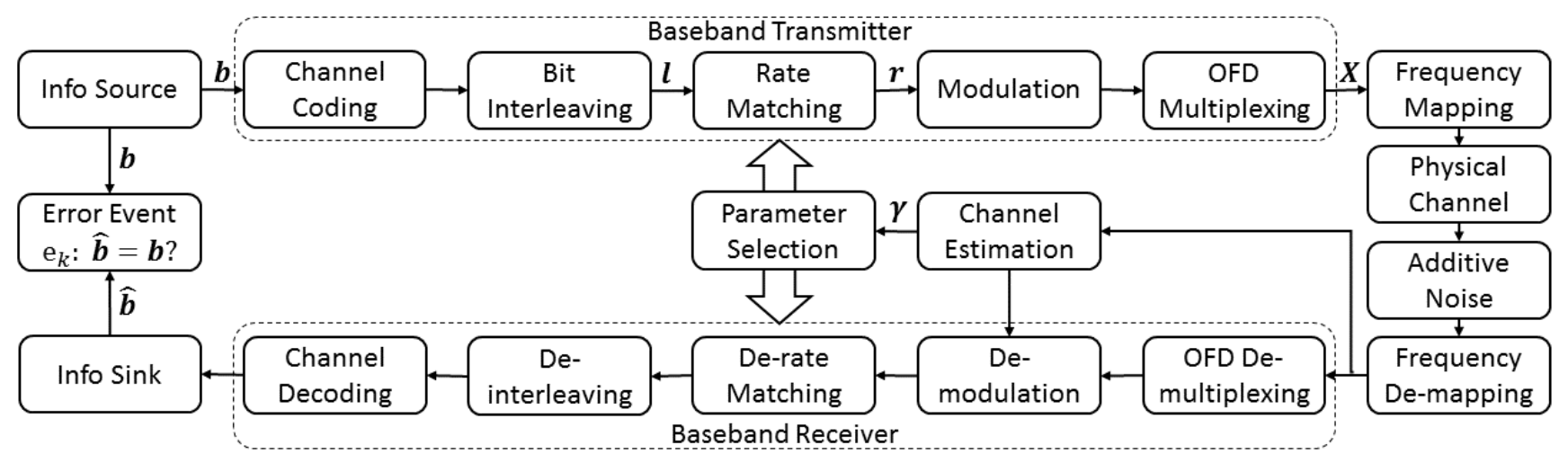

Fig. 1. Block diagram of the BICM-OFDM link chain considered in this paper. The parameter selection module exploits knowledge of the instantaneous channel state to select one out of several possible transmission parameter configurations in each frame.

leaved bits are then used to generate the "rate-matched" bit sequence $\boldsymbol{r}=\left(r_{1}, \ldots, r_{M S J}\right)$ according to $r_{i}=l_{i \bmod L}, i=1, \ldots, M S J$, where $M$ is the number of transmission subcarriers, $S$ is the number of frame OFDM symbols, and $J$ is the modulation order. The channel code rate is therefore $\mathcal{R}=T / M S J$. The rate matched bits are mapped onto $M S$ modulated symbols by a labeling function that assigns one out of $2^{J}$ complex-valued constellation symbols to each $J$-tuple of bits. Finally, a length- $M$ IFFT operation is applied on each group of $M$ modulated symbols to generate the frame OFDM symbols $\boldsymbol{X}=\left(\boldsymbol{x}_{1}, \ldots, \boldsymbol{x}_{S}\right)$ and mapped onto physical resources.

The frame OFDM symbols are transmitted over the physical channel resulting in the received signal $\boldsymbol{y}_{s}=\boldsymbol{h} \odot \boldsymbol{x}_{s}+\boldsymbol{g}_{s}, s=$ $1, \ldots, S$, where $\odot$ denotes the Hadamard product, $\boldsymbol{h}$ is the complexvalued vector of channel coefficients in the frequency domain, and $\boldsymbol{g}_{s} \sim \mathcal{N}^{M \times 1}\left(0, \sigma^{2}\right)$ is i.i.d. noise. We assume that the channel vector remains constant for the frame OFDM symbols (i.e., the channel is block fading). At the receiver, each received OFDM symbol $\boldsymbol{y}_{s}$ is multiplied by the elementwise inverse of the estimated frequency-domain channel vector, followed by a length-M FFT operation for OFDM de-multiplexing. The de-multiplexed symbols are then mapped onto soft values through an inverse labeling operation, de-interleaved, and decoded by the channel decoder to generate the reconstructed bit sequence $\hat{\boldsymbol{b}}=\left(\hat{b}_{1}, \ldots, \hat{b}_{T}\right)$. We define the binary frame error event at the receiver as

$$
e=\left\{\begin{array}{lll}
0 & \text { if } \quad \hat{\boldsymbol{b}}=\boldsymbol{b} \\
1 & \text { if } \quad \hat{\boldsymbol{b}} \neq \boldsymbol{b}
\end{array} .\right.
$$

\subsection{Estimation}

The BICM-OFDM link chain described above can be approximated as a stochastic non-linear function that generates a frame error event with an unknown probability distribution for the frame channel state and a particular choice of transmission parameters. In Fig. 2, we illustrate an abstract model of the BICM-OFDM link chain, which is parameterized by the model parameters $\boldsymbol{\theta}$ and maps the observed channel state characterized by the received per-subcarrier channel SINRs, $\gamma=\left(\gamma_{1} \ldots, \gamma_{M}\right)$, to the observed frame error event, .i.e.,

$$
P_{E_{k} \mid \Gamma}\left(e_{k} \mid \boldsymbol{\gamma} ; \boldsymbol{\theta}\right)=\rho_{k}^{e_{k}}\left(1-\rho_{k}\right)^{1-e_{k}}
$$

where the $k \in 1, \ldots, K$ denotes the $k^{\text {th }}$ transmission parameter configuration. Here, $\rho_{k}=\rho_{k}(\boldsymbol{\gamma} ; \boldsymbol{\theta})=P_{E_{k} \mid \Gamma}\left(E_{k}=1 \mid \boldsymbol{\gamma} ; \boldsymbol{\theta}\right)$ is the conditional frame error probability (FEP). In the rest of this section, we show that the ML estimator of the model parameters asymptotically estimates the true conditional FEPs.

The ML estimator [11] of the model parameters for $n=$ $1, \ldots, N$ frame realizations is defined as

$$
\begin{aligned}
\hat{\boldsymbol{\theta}}^{\mathrm{ML}} & =\arg \max _{\hat{\boldsymbol{\theta}}} \sum_{k=1}^{K} \prod_{n=1}^{N} P_{E_{k}, \Gamma}\left(e_{k}^{n}, \boldsymbol{\gamma}^{n} ; \hat{\boldsymbol{\theta}}\right) \\
& =\arg \max _{\hat{\boldsymbol{\theta}}} \int \sum_{k=1}^{K} \sum_{n=1}^{N} \ln P_{E_{k} \mid \Gamma}\left(e_{k}^{n} \mid \boldsymbol{\gamma}^{n} ; \hat{\boldsymbol{\theta}}\right) P_{\Gamma}(\boldsymbol{\gamma}) d \boldsymbol{\gamma} \\
& \triangleq \arg \max _{\hat{\boldsymbol{\theta}}} \mathcal{C}(\hat{\boldsymbol{\theta}}), \text { where } \\
\mathcal{C}(\hat{\boldsymbol{\theta}}) & =\sum_{k=1}^{K}\left(\frac{1}{N} \sum_{n=1}^{N} \ln P_{E_{k} \mid \Gamma}\left(e_{k}^{n} \mid \boldsymbol{\gamma}^{n} ; \hat{\boldsymbol{\theta}}\right)\right)
\end{aligned}
$$

is the cost function to be maximized, and we have used the fact that the channel state is independent of the model. In the limit of infinite training samples, it follows by the law of large numbers that

$$
\mathcal{C}(\hat{\boldsymbol{\theta}}) \stackrel{N \rightarrow \infty}{\longrightarrow} \sum_{k=1}^{K} E\left\{\ln P\left(E_{k} \mid \boldsymbol{\Gamma} ; \hat{\boldsymbol{\theta}}\right)\right\}
$$

where $P\left(E_{k} \mid \boldsymbol{\Gamma} ; \hat{\boldsymbol{\theta}}\right) \triangleq P_{E_{k} \mid \boldsymbol{\Gamma}}\left(E_{k} \mid \boldsymbol{\Gamma} ; \hat{\boldsymbol{\theta}}\right)$ for brevity, and where the expectation is taken over $P\left(E_{k}, \boldsymbol{\Gamma} ; \boldsymbol{\theta}\right)$. We now subtract and add the true pmf to the r.h.s. of Eq. 5 to obtain

$$
\begin{aligned}
\mathcal{C}(\hat{\boldsymbol{\theta}}) & =\sum_{k=1}^{K} E\left\{\ln P\left(E_{k} \mid \boldsymbol{\Gamma} ; \hat{\boldsymbol{\theta}}\right)-\ln P\left(E_{k} \mid \boldsymbol{\Gamma} ; \boldsymbol{\theta}\right)+\ln P\left(E_{k} \mid \boldsymbol{\Gamma} ; \boldsymbol{\theta}\right)\right\} \\
& =-\sum_{k=1}^{K} E\left\{\ln \frac{P\left(E_{k} \mid \boldsymbol{\Gamma} ; \boldsymbol{\theta}\right)}{P\left(E_{k} \mid \boldsymbol{\Gamma} ; \hat{\boldsymbol{\theta}}\right)}\right\}+\sum_{k=1}^{K} E\left\{\ln P\left(E_{k} \mid \boldsymbol{\Gamma} ; \boldsymbol{\theta}\right)\right\} .
\end{aligned}
$$

The second term in 6 is independent of the argument to be maximized. Further we observe that by multiplying and dividing the first term with probability distribution $P_{\Gamma}(\gamma)$, we obtain

$$
\begin{aligned}
& \sum_{k=1}^{K} E\left\{\ln \frac{P\left(E_{k} \mid \boldsymbol{\Gamma} ; \boldsymbol{\theta}\right) P(\boldsymbol{\Gamma})}{P\left(E_{k} \mid \boldsymbol{\Gamma} ; \hat{\boldsymbol{\theta}}\right) P(\boldsymbol{\Gamma})}\right\}=\sum_{k=1}^{K} E\left\{\ln \frac{P\left(E_{k}, \boldsymbol{\Gamma} ; \boldsymbol{\theta}\right)}{P\left(E_{k}, \boldsymbol{\Gamma} ; \hat{\boldsymbol{\theta}}\right)}\right\}= \\
& \sum_{k=1}^{K} \mathrm{KL}\left\{P\left(E_{k} \mid \boldsymbol{\Gamma} ; \boldsymbol{\theta}\right) \| P\left(E_{k} \mid \boldsymbol{\Gamma} ; \hat{\boldsymbol{\theta}}\right)\right\}
\end{aligned}
$$




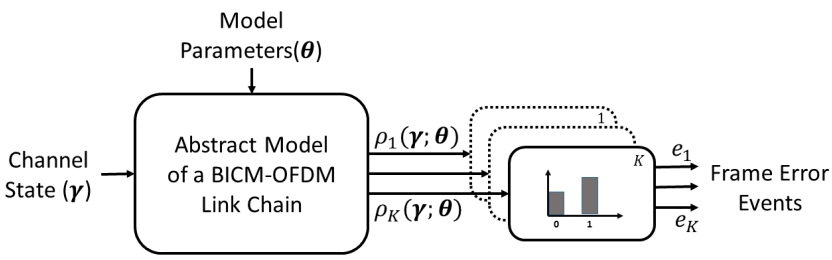

Fig. 2. Abtract model of a BICM-OFDM link chain that maps the observed channel state to the frame error events for $k=1, \ldots, K$ transmission parameter configurations.

where $\mathrm{KL}(\cdot \| \cdot)$ is the Kullback-Liebler divergence (KLD) between the true and estimated pmfs. Given that the KLD is non-negative, and equal to zero if and only if $P\left(E_{k} \mid \boldsymbol{\Gamma} ; \boldsymbol{\theta}\right)=P\left(E_{k} \mid \boldsymbol{\Gamma} ; \hat{\boldsymbol{\theta}}\right)$, it follows that the ML estimator converges to the true FEP distribution in the limit of large $N$. Note however that $P\left(E_{k} \mid \boldsymbol{\Gamma} ; \boldsymbol{\theta}\right)=P\left(E_{k} \mid \boldsymbol{\Gamma} ; \hat{\boldsymbol{\theta}}\right)$ does not necessarily imply that $\hat{\boldsymbol{\theta}}^{\mathrm{ML}}=\boldsymbol{\theta}$ as the ML estimate of $\boldsymbol{\theta}$ may be non-unique.

\section{FEP PREDICTION TECHNIQUES}

\subsection{Effective SINR Approach}

In this subsection we outline the widely studied exponential effective SIR metric (EESM) approach, where the the channel state characterized by the per-subcarrier SINRs is compressed to a scalar "effective" SINR for an equivalent AWGN channel [8]. The FEP for the $k^{t h}$ transmission parameter configuration is then predicted to be

$$
\begin{aligned}
\hat{\rho}_{k}^{\mathrm{ESM}}(\gamma) & =\rho_{k}^{\mathrm{AWGN}}\left(g_{k}(\gamma)\right), \\
\text { where } g_{k}(\gamma) & =\beta_{k} \log \left(\frac{1}{M} \sum_{m=1}^{M} \exp \left(\frac{\gamma_{m}}{\beta_{k}}\right)\right)
\end{aligned}
$$

is the EESM for channel state $\gamma$, and $\beta_{k}$ is a tunable parameter. For the frequency selective channels commonly observed in practical systems, $g_{k}(\gamma)$ amounts to a lossy compression of the channel state vector, since the original channel state can no longer be recovered. The FEP for the equivalent AWGN channel, $\rho_{k}^{\mathrm{AWGN}}\left(g_{k}(\gamma)\right)$, is obtained by interpolating between several Monte Carlo simulated FEP values for the AWGN channel. The optimal values of the tuning parameters $\beta_{k}$ can be determined by minmizing the Euclidean distance between the predicted FEP and observed frame errors for $n=1, \ldots, N$ training frames, i.e.,

$$
\beta_{k}^{\text {opt }}=\arg \min _{\beta_{k}} \sum_{n=1}^{N}\left|\hat{\rho}_{k}^{\text {n, ESM }}-e_{k}^{n}\right|^{2} .
$$

As $N$ grows to infinity, $\beta_{k}^{\text {opt }}$ also minimizes the least squares error between the estimated and true FEPs, however, we skip the proof here owing to space constraints.

\subsection{Deep Learning Approach}

The EESM approach described earlier relies on a scalar approximation of the channel state, which is obtained through a lossy compression and therefore does not guarantee optimality of the the corresponding FEP prediction. In this subsection we describe our approach for FEP prediction based on deep neural networks, which discriminatively learns the mapping between the (uncompressed) channel state vector and the corresponding FEPs for multiple transmission parameter configurations.
Neural networks have long been known as a powerful tool for approximating a wide range of highly non-linear functions, however, their acceptance for implementation in practical systems has been limited by an insufficient understanding of the models that they learn from training data. Although a complete understanding of neural networks is still a topic of active research, several recent breakthroughs related to deep neural networks coupled with cheap computational power have led to drastic performance improvements for several challenging problems [12]. In this paper, we consider the fully connected $L$-layered deep neural network illustrated in Fig. 3 , for which the output of the $l^{t h}$ "hidden layer" with dimension $d_{l}$ can be described as

$$
\boldsymbol{\eta}^{(l)}=\phi^{(l)}\left(\boldsymbol{W}^{(l)} \boldsymbol{\eta}^{(l-1)}+\boldsymbol{b}^{(l)}\right),
$$

where $\boldsymbol{W}^{(l)}$ is the trainable $d_{l-1} \times d_{l}$ weight matrix, $\boldsymbol{b}^{(l)}$ is the trainable $d_{l} \times 1$ bias vector, and $\phi^{(l)}$ is a fixed non-linear "activation" function. By simply substituting the system parameters $\boldsymbol{\theta}$ with neural network weights and biases, we allow the neural network to learn the set of mappings $\rho_{k}(\boldsymbol{\gamma} ; \hat{\boldsymbol{\theta}})$ for $k=1, \ldots, K$ from data.

It has been shown previously that an ordering of the persubcarrier SINRs can be used to sufficiently parameterize the frame error rate while reducing the training requirements [9]. Therefore in this paper, we use the sorted per-subcarrier SINR vector, $\widetilde{\gamma}$, as the input to the network, i.e., $\boldsymbol{\eta}^{(0)} \triangleq \widetilde{\gamma}=\left(\widetilde{\gamma}_{1}, \ldots, \widetilde{\gamma}_{M}\right)$. The activation function for the each of the non-output layers can be any continuously differentiable non-linear function within some practical constraints [12]. For the output layer, we choose sigmoid activation function $\phi^{(L)}(x)=1 /\left(1+e^{-x}\right)$ and interpret the network outputs as the predicted FEPs, i.e., $\boldsymbol{\eta}^{(L)} \triangleq \hat{\boldsymbol{\rho}}^{\mathrm{NN}}=\left(\hat{\rho}_{1}^{\mathrm{NN}}, \ldots, \hat{\rho}_{K}^{\mathrm{NN}}\right)$. We choose the cross-entropy loss between the network outputs and the frame error events as the cost function, i.e.,

$$
\begin{aligned}
\mathcal{C}(\hat{\boldsymbol{\theta}}) & =\frac{1}{K} \sum_{k=1}^{K} e_{k} \ln \hat{\rho}_{k}^{\mathrm{NN}}+\left(1-e_{k}\right) \ln \left(1-\hat{\rho}_{k}^{\mathrm{NN}}\right) \\
& =\frac{1}{K} \sum_{k=1}^{K} \ln P_{E_{k} \mid \boldsymbol{\Gamma}}\left(e_{k} \mid \boldsymbol{\gamma} ; \hat{\boldsymbol{\theta}}\right),
\end{aligned}
$$

where the latter expression is obtained using Eq. 27. By observing the direct correspondence between Eqs. (4) and (13), we observe that minimizing the neural network cost function over $n=1, \ldots, N$ training frames is equivalent to ML estimation of the neural network parameters, which we have shown to estimate the true FEPs.

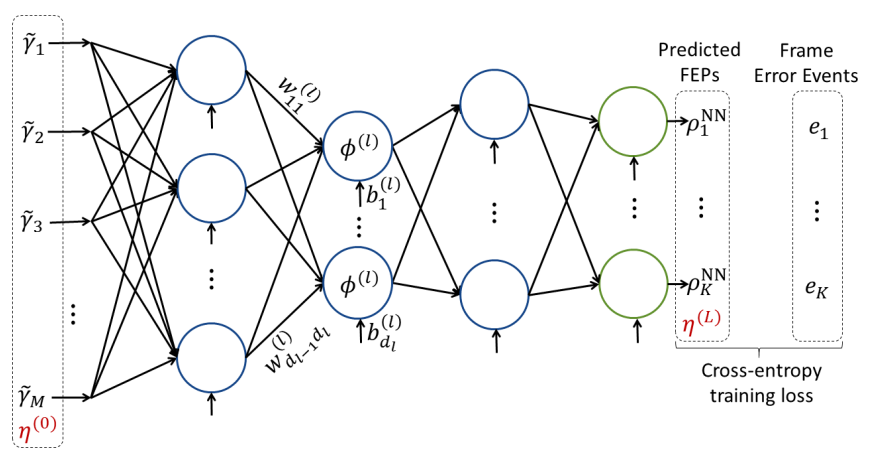

Fig. 3. Neural network layout that maps the channel state characterized by per-subcarrier SINR values to the FEP for each of the $K$ transmission parameter configurations. 
In is crucial to point out that there is no guarantee that the neural network trained using stochastic gradient decent will converge to a ML estimates of the parameters, however, our simulation results indicate that the neural network does indeed provide good estimates of $\rho_{k}(\boldsymbol{\gamma} ; \hat{\boldsymbol{\theta}})$. Further, the result obtained above is equivalent to the result derived in the context of cross entropy cost function in [13]. However, we believe that it is instructive to demonstrate this result in the context of ML estimation.

\subsection{FEP Prediction for Throughput Maxmization}

The FEP prediction studied in this paper can be used to select the transmission parameters in each frame to optimize one or more desired link metrics. In this paper, we study the selection of the optimal channel code rate $\mathcal{R}_{k}, k \in\{1, \ldots, K\}$ that maximizes the link throughput over a fading channel, while rest of the transmission parameters are kept fixed. For this, the channel code rate that maximizes the predicted expected throughput in that frame is selected for each approach, i.e., $k^{\mathrm{EESM}}=\arg \max _{k} T_{k}\left(1-\rho_{k}^{\mathrm{EESM}}\right)$, and $\mathrm{NN}=\arg \max _{k} T_{k}\left(1-\rho_{k}^{\mathrm{NN}}\right)$ for the EESM and deep neural network approaches respectively, where $T_{k}=M S J \mathcal{R}_{k}$ is the number of trasnmitted information bits when the $k^{\text {th }}$ channel code rate is selected. The realized throughput over $N$ evaluted frames is therefore $\mathcal{T}^{\mathrm{EESM}}=\frac{1}{N} \sum_{n} T_{k^{\mathrm{EESM}}}^{n}\left(1-e_{k \mathrm{EESM}}^{n}\right)$ and $\mathcal{T}^{\mathrm{NN}}=\frac{1}{N} \sum_{n} T_{k^{\mathrm{NN}}}^{n}(1-$ $\left.e_{k^{\mathrm{NN}}}^{n}\right)$ respectively, where $e_{k}^{n}$ denotes the actual frame error event for the $k^{\text {th }}$ channel code rate in the $n^{\text {th }}$ frame.

\section{NUMERICAL RESULTS}

In this section we provide simulation results for the FEP prediction accuracy and the achieved link throughput for our proposed deep learning approach and contrast it with the EESM approach results. The simulation parameters are listed in Table 1 We use Python wrappers over the IT++ library to generate the results described in this section, which allows easy integration of Tensorflow for the neural network implementation and evaluations [14] [15]. We assume perfect knowledge of the channel at the transmitter as well as the receiver, i.e., $\gamma=|\boldsymbol{h}|^{2} / \sigma^{2}$, and use a rate- $1 / 3$ Turbo channel encoder. The neural network comprises 3 hidden layers with dimensions $\{60,10,60\}$ respectively and each hidden layer employs a Rectified Linear Unit (ReLU) activation function [12]. The training datasets for EESM and neural network approaches are generated using $10^{4}$ frames for each channel code rate. The test dataset for throughput maximization comprises $10^{3}$ realizations for 10 evenly spaced long term average SINR values in the range $[-10,20) \mathrm{dB}$.

Table 1. Simulation Parameters

\begin{tabular}{|l|c|}
\hline Simulation Parameter & Value \\
\hline Channel Model & EPA \\
Number of Frame OFDM Symbols $(S)$ & 12 \\
Number of Transmit Subcarriers $(M)$ & 600 \\
Modulation Order $(J)$ & 2 \\
Channel Code Rates $\left(\mathcal{R}_{k}\right)$ & {$[0.01,0.02 \ldots, 0.30]$} \\
\hline
\end{tabular}

The Root Mean Square Error (RMSE) for FEP prediction performance over is shown in Fig. 4 . We observe that the neural network approach outperforms the EESM approach in terms of FEP prediction performance. The throughput performance of the EESM and neural network approaches is shown in Fig. 5 along with the upper bound "Genie" throughput curve that is obtained by exhaustively searching the maximum achieved throughput in each frame.
We observe that our approach increases the throughput compared to the EESM approach.

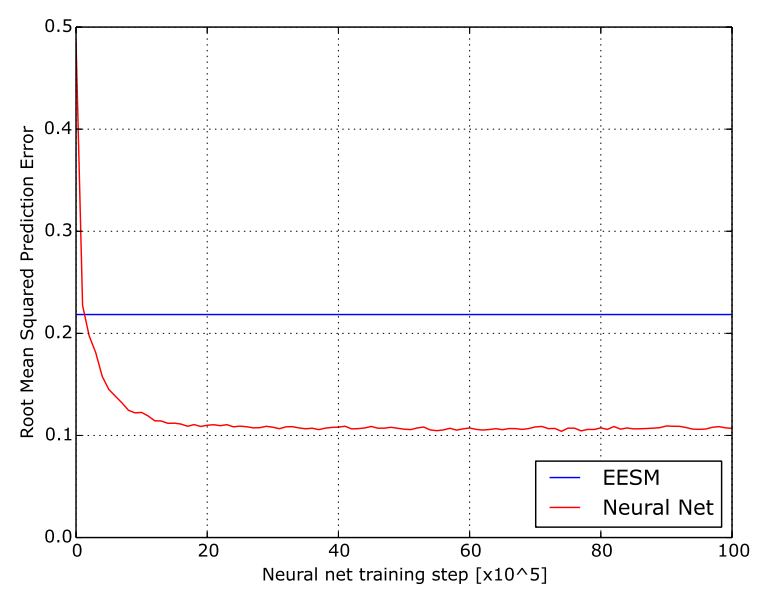

Fig. 4. RMSE of the FEP prediction error using EESM and Neural Network approaches.

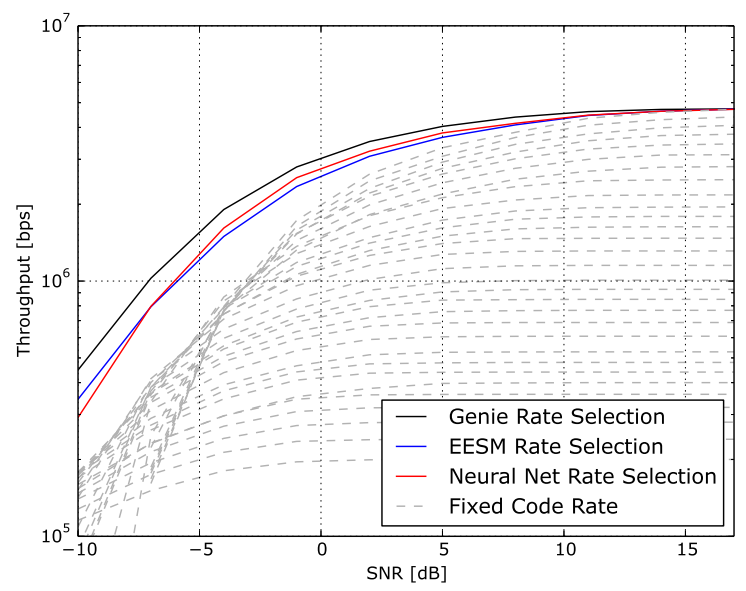

Fig. 5. Achieved throughput with Genie, EESM and Neural Network rate selection for optimal channel code rate selection.

\section{CONCLUSIONS}

In this paper we have proposed a deep learning approach for FEP prediction which directly learns the mapping between the highdimensional channel state characterized by the per-subcarrier SINRs and the FEPs for an arbitrary set of transmission configurations. Further, by utilizing a training scheme that relies only on the oberved channel state and the binary frame error events, our approach is able to estimate the true FEPs. Finally, we have shown that by exploiting better FEP predictions from our approach, it is possible to increase the realized link throughput by optimally selecting the channel code rate in each frame. 


\section{REFERENCES}

[1] A. Molisch, Wireless Communications. Wiley, 2010.

[2] G. Caire, G. Taricco, and E. Biglieri, "Bit-interleaved coded modulation," IEEE Transactions on Information Theory, vol. 44, no. 3, pp. 927-946, May 1998.

[3] D. Agrawal, V. Tarokh, A. Naguib, and N. Seshadri, "Spacetime coded OFDM for high data-rate wireless communication over wideband channels," in Vehicular Technology Conference, 1998. VTC 98. 48th IEEE, vol. 3, May 1998, pp. 2232-2236 vol.3.

[4] A. Zaidi, R. Baldemair, M. Andersson, S. Faxér, V.-M. Casés, and Z. Wang, "Designing for the future: the 5G NR physical layer," Ericsson Technology Review, July 2017.

[5] S. Nanda and K. M. Rege, "Frame error rates for convolutional codes on fading channels and the concept of effective $E_{b} / N_{0}$," IEEE Transactions on Vehicular Technology, vol. 47, no. 4, pp. 1245-1250, Nov 1998.

[6] Y. W. Blankenship, P. J. Sartori, B. K. Classon, V. Desai, and K. L. Baum, "Link error prediction methods for multicarrier systems," in IEEE 60th Vehicular Technology Conference, 2004. VTC2004-Fall. 2004, vol. 6, Sept 2004, pp. 4175-4179.

[7] Ericsson, "Effective SNR mapping for modelling frame error rates in multiple-state channels," Tech. Rep. 3GPP2-C3020030429-010, April 2003.

[8] K. Brueninghaus, D. Astely, T. Salzer, S. Visuri, A. Alexiou, S. Karger, and G. A. Seraji, "Link performance models for system level simulations of broadband radio access systems," in 2005 IEEE 16th International Symposium on Personal, Indoor and Mobile Radio Communications, vol. 4, Sept 2005, pp. 2306-2311.

[9] R. C. Daniels, C. M. Caramanis, and R. W. Heath, "Adaptation in Convolutionally Coded MIMO-OFDM Wireless Systems Through Supervised Learning and SNR Ordering," IEEE Transactions on Vehicular Technology, vol. 59, no. 1, pp. 114126, Jan 2010.

[10] S. Yun and C. Caramanis, "Multiclass support vector machines for adaptation in MIMO-OFDM wireless systems," in 2009 47th Annual Allerton Conference on Communication, Control, and Computing (Allerton), Sept 2009, pp. 1145-1152.

[11] S. M. Kay, Fundamentals of Statistical Signal Processing: Estimation Theory. Prentice Hall PTR, 1993.

[12] I. Goodfellow, Y. Bengio, and A. Courville, Deep Learning. MIT Press, 2016.

[13] M. D. Richard and R. P. Lippmann, "Neural Network Classifiers Estimate Bayesian a Posteriori Probabilities," Neural Computation, vol. 3, no. 4, pp. 461-483, 1991.

[14] M. Abadi, A. Agarwal, P. Barham, E. Brevdo, Z. Chen, C. Citro, G. S. Corrado, A. Davis, J. Dean, M. Devin, S. Ghemawat, I. Goodfellow, A. Harp, G. Irving, M. Isard, Y. Jia, R. Jozefowicz, L. Kaiser, M. Kudlur, J. Levenberg, D. Mané, R. Monga, S. Moore, D. Murray, C. Olah, M. Schuster, J. Shlens, B. Steiner, I. Sutskever, K. Talwar, P. Tucker, V. Vanhoucke, V. Vasudevan, F. Viégas, O. Vinyals, P. Warden, M. Wattenberg, M. Wicke, Y. Yu, and X. Zheng, "TensorFlow: Large-scale machine learning on heterogeneous systems," 2015, software available from tensorflow.org. [Online]. Available: http://tensorflow.org/

[15] "PY_ITPP: Python wrappers over IT++." 